\title{
Stress Response Analysis of Metallic Thin-walled Stiffened Structure under Thermo-acoustic Loadings
}

\author{
Yundong Sha ${ }^{1}$, Xiaoning Tang $^{1, a}$, Jian Wang $^{1}$, Jinzhuo Jiang ${ }^{2}$, Fengtong Zhao ${ }^{1}$ \\ ${ }^{1}$ Shenyang Aerospace University, Liaoning Key Laboratory of Digital Technology Simulation and \\ Test Techniques, Liaoning, Shenyang, 110136, P. R. China \\ ${ }^{2}$ AVIC Shenyang Liming Aero-Engine Group Corporation LTD, Liaoning, Shenyang, 110136, P. R. \\ China
}

a894334790@qq.com

\begin{abstract}
Keywords: Thin-walled stiffened structure, Acoustic loads, Uniform temperature field, Stress dynamic response.
\end{abstract}

\begin{abstract}
For the realization of the aircraft to reduce weight and improve efficiency, thin-walled structure is widely used in aerospace vehicle. Thin-walled structure under the extremely harsh environment- high temperature and high acoustic loading- can cause strong nonlinear large deflection vibration characteristics, which leads to structural fatigue failure occur prematurely. According to the basic theory of finite element, this paper calculated the dynamic response of thin-walled stiffened structures in uniform temperature field under the gaussian white noise loading. According to the calculated stress, the thin-walled stiffened structure stress characteristics are analyzed.
\end{abstract}

\section{Introduction}

New hypersonic flight vehicle structure facing increasingly severe load conditions, the damage of the structure under external load acoustic are increasingly obvious. As the same time, the material properties, dynamics, automatic control and so on can be serious influenced when high temperature acting on the structure ${ }^{[1]}$. Therefore, the thermal-acoustic loading problem must be taken into account on the design of new aircraft structure.

Foreign research institutions were studied separately thermo-acoustic fatigue test of overall structure, as well as the typical structural components ${ }^{[2]}$. Early nineties, NASA Langley Centre carried out the thermal-acoustic experiment, one part to research the test technology of superalloys thin-walled honeycomb structure to analyze dynamic response. For the issue, strain response was tested separately at room temperature and high temperature, high acoustic energy and broadband loadings. In another part, the plate and stiffener thin-walled stiffened structures for the study, test specimen under the incentive of $160 \mathrm{~dB}$, analyze the acoustic fatigue characteristics ${ }^{[3]}$.

After the 21st century, the problem of "the next generation of launch technology (NGLT)" is came up with United States National Aeronautics and Space Administration (NASA), a prelude to structural design and thermal design which gradually shifted to the thermal structure design for advanced anti-aircraft that did just that, ,also carried out series of thermal-acoustic test project . Thermal noise test expensive and not easy to get reliable data, therefore, in the course of study thermo-acoustic problem, the theoretical analysis and numerical study was highly valued ${ }^{[4]}$. Accurate and reliable predict the nonlinear dynamic response of structural components under the thermo-acoustic environment has become an important challenge.

Professor Yundong Sha, Shenyang Aerospace University ${ }^{[5,6]}$, made a series of studies of the thin-walled fatigue life under the thermal-acoustic loadings. Currently dynamic response and fatigue life estimate of metal, composite structures under the thermo-acoustic are under studying. In this paper, finite element model of thin-walled stiffened structures were established by FEM analysis, applying Gaussian white noise loads and uniform temperature on the thin-walled stiffened structures, calculated represents nodes dynamic stress, analyze the stress characteristics of structural. 


\section{Theory analysis}

\section{Von Karman large deformation equation for plates}

The random pressure, inertia force and damping force have been known, they are $p(x, y, t)$, $\rho h \frac{\partial^{2} w}{\partial t^{2}}, \quad c \frac{\partial w}{\partial t}$. According to balance of forces, the balance between the torque can be exported wall thermal acoustic loading large deflection nonlinear equations of motion under the combined effect of the following

$$
\begin{aligned}
& \rho h \frac{\partial^{2} w}{\partial t^{2}}+c \frac{\partial w}{\partial t}+D \nabla^{4} w+2 \frac{\partial^{2} F}{\partial x \partial y} \frac{\partial^{2} w}{\partial x \partial y}-\frac{\partial^{2} F}{\partial y^{2}} \frac{\partial^{2} w}{\partial x^{2}}-\frac{\partial^{2} F}{\partial x^{2}} \frac{\partial^{2} w}{\partial y^{2}}+D \alpha(1+\mu) \nabla^{2} \theta \\
& =p(x, y, t)
\end{aligned}
$$

where $\nabla^{4}=\nabla^{2} \cdot \nabla^{2}=\left(\frac{\partial^{2}}{\partial x^{2}}+\frac{\partial^{2}}{\partial y^{2}}\right)^{2}, \nabla^{4}$ is bi-harmonic operator.

\section{FEM theory analysis}

FEM equations of motion can be expressed as follows

$$
M\{\ddot{w}\}+\left[K-K_{T}+K 1(w)+K 2(w, w)\right]\{w\}=\{P\}+\left\{P_{T}\right\} .
$$

where $M$ is the mass matrix, $K$ is the linear stiffness matrix, $w$ is the nodal displacement time-related, $\{P\}$ is the nodal force vector of time-related. $K 1$ and $K 2$ are first-order and second-order nonlinear stiffness matrix. $K_{T}$ is the linear stress stiffness matrix caused by the thermal stress. $\left\{P_{T}\right\}$ is the equivalent nodal force vector caused by the Thermal stress. Use Newton-Raphson iteration method and Newmark time domain integral method for solving equations, obtained by the displacement of the panel node. The resulting displacement into the strain equation and the surface curvature equation to give the plane strain and curvature of the panel, and then into to the equation, siding the s plane train of the panel into the equation, which can be obtained in response to panel stress .

\section{Numerical Analysis}

In this paper, cross rib thin-walled structure as the research object, using Clamped constraints. Based on the basic theory of finite element method, using the whole modal transient dynamics calculation method, geometric nonlinearity of the cross rib thin-walled structures is taken into consideration, calculate the dynamic stress of cross rib thin-walled structures under thermal-acoustic loading. The Gaussian white acoustic loading is applied which is uniform space and the cut-off frequency at $500 \mathrm{~Hz}$. Sound pressure level in the range from SPL $=140 \mathrm{~dB}$ to $\mathrm{SPL}=170 \mathrm{~dB}$. Cross rib thin-walled structure shown in Figure 2, the material parameters as shown in Table 1.

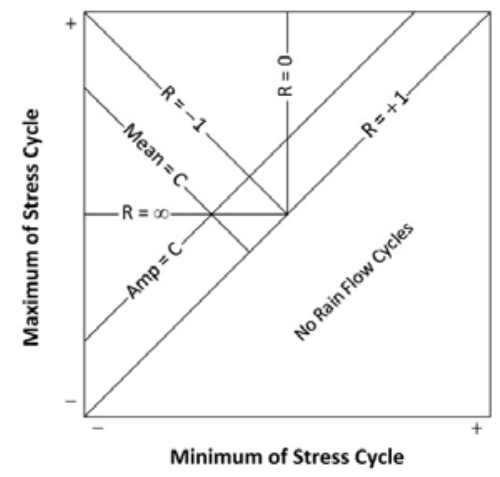

Figure 1 WAFO rain flow matrix

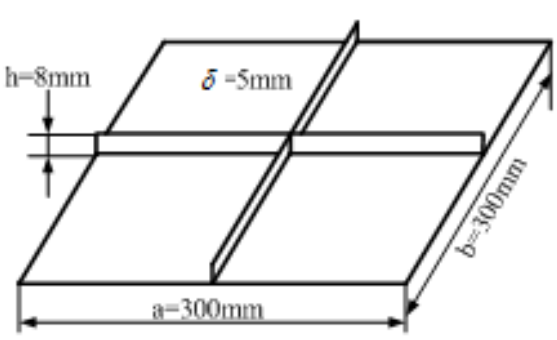

Figure 2 The dimensions of the thin-walled structure 
Table 1 Material properties of GH536

\begin{tabular}{|c|c|c|c|c|c|}
\hline $\mathrm{T}\left[{ }^{\circ} \mathrm{C}\right]$ & $\rho\left[\mathrm{Kg} / \mathrm{m}^{3}\right]$ & $\mu$ & $\alpha\left[\mathrm{K}^{-1}\right]$ & $\mathrm{E}[\mathrm{GPa}]$ & $\begin{array}{c}\text { Thermal Conductivity } \\
{\left[\mathrm{W} / \mathrm{m}^{\circ}{ }^{\circ} \mathrm{C}\right]}\end{array}$ \\
\hline 0 & 8230 & 0.33 & $1.27 \times 10^{-6}$ & 177 & 8 \\
\hline 200 & 8230 & 0.33 & $14 \times 10^{-6}$ & 170 & 10.4 \\
\hline 400 & 8230 & 0.33 & $15.5^{\times 10^{-6}}$ & 159 & 14 \\
\hline 600 & 8230 & 0.32 & $17.4 \times 10^{-6}$ & 145 & 18 \\
\hline 800 & 8230 & 0.32 & $19.1 \times 10^{-6}$ & 131 & 21.6 \\
\hline
\end{tabular}

In order to investigate the effect of temperature loadings on stiffened plate response, the buckling coefficient $\mathrm{S}$ is introduced. $S=T / T_{c r}$ Where $T$ is the temperature of uniform temperature field, and $T_{c r}$ denotes the first order thermal buckling critical temperature under the uniform steady state thermal loading. Calculation shows that the first order thermal buckling critical temperature is $92.846{ }^{\circ} \mathrm{C}$.The intersection point of reinforcement grid was selected as a typical node.The SPL is fixed in $170 \mathrm{~dB}, \mathrm{X}$-component of stress of the typical node was shown in figure 3.

We can know from the figures that when the buckling coefficient is relatively small, stiffened plate has a random vibration with the small amplitude in the initial equilibrium position.Until the temperature coefficient is reaching 0.9 , the vibration of stiffened plate will become severe with the increasing amplitude,occurring consecutive snap-through phenomenon and changing the equilibrium position in a small scale.With the persistently increase of temperature and the temperature coefficient is reaching 1.4 ,the stiffened plate will be in thermal post-buckling region,vibrating around the other equilibrium position and decreasing vibration amplitude slightly. This series of changes above clearly explain the large deflection nonlinear process of stability, instability and stability, which happened in the process of thermal-buckling .

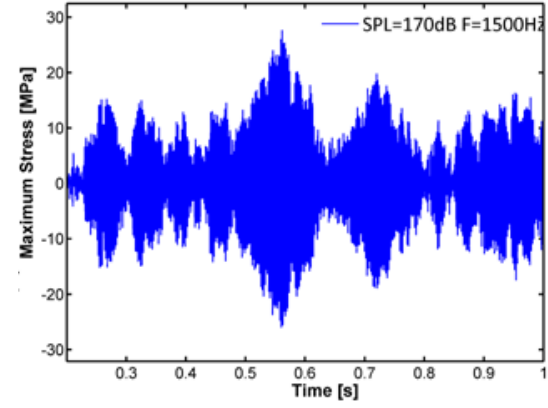

(a) X-component of stress $(S=0)$

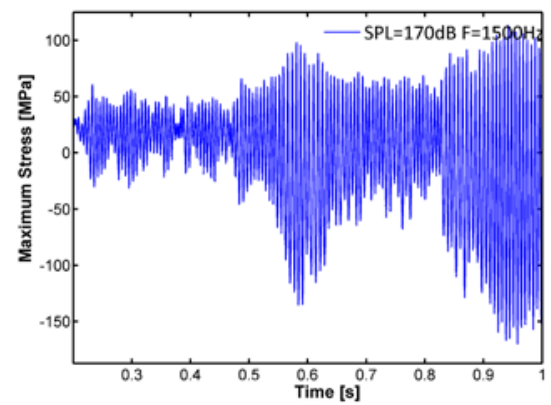

(c) X-component of stress $(S=0.9)$

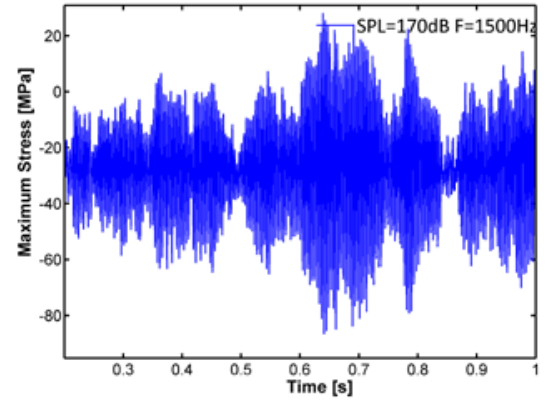

(b) X-component of stress $(S=0.6)$

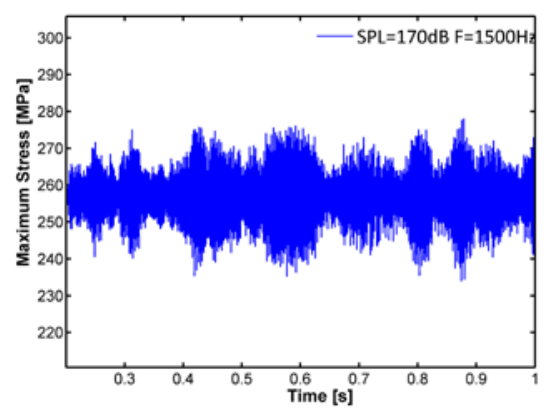

(d) X-component of stress $(S=1.4)$

Figure 3 Nonlinear dynamic stress of the thin-walled structure (SPL=170dB)

The displacement power spectral density (PSD) of typical node with different combinations of thermal-acoustic loadings are shown in figure 4.We know that the displacement response of PSD change with thermal loading from figure 4(a) and 4(b) .Consider the fundamental frequency of the critical buckling as a criterion.In the pre-buckled region,the fundamental frequency decreased with 
the increase of the buckling coefficient $S$, reducing the stiffness of structure .And in the post-buckled region the fundamental frequency increased with the increase of the buckling coefficient S,rising the stiffness of structure.

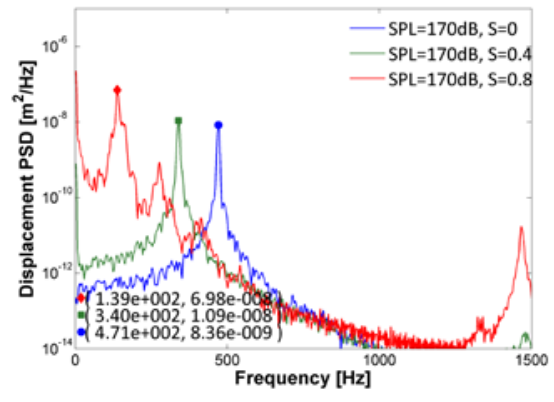

(a) pre-buckling

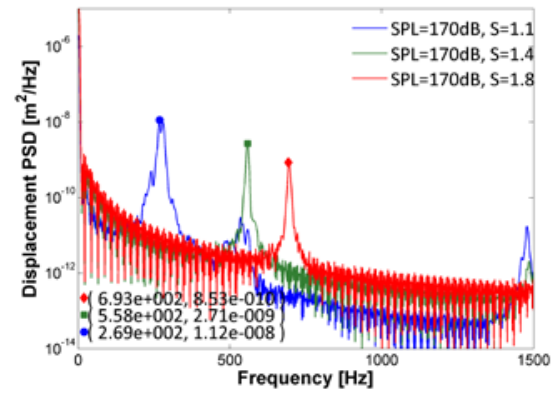

(b) post-buckling

Figure 4 Displacement PSD under different temperature field (SPL=170)

\section{Summary}

(1) In the dynamic response calculation of cross rib thin-walled stiffened structure with four clamped edges suffering from thermal-acoustic loadings, the results show that when acoustic excitation is a constant, the equilibrium position of the stiffened plate vibration may change with the temperature.

(2) Temperature field has a significant influence on displacement and the mean value of stress response of structure. Thermal buckling equilibrium position of the stiffened plate will be changed from pre-buckled state to post-buckled state, vibrating around the two thermal buckling equilibrium positions. Once the temperature is closing to the buckling temperature, there exists persistent snap-through phenomenon.

(3) In pre-buckled region, the fundamental frequency of the cross rib thin-walled stiffened structure decreased gradually with the increase of temperature. In post-buckled region, The fundamental frequency of the cross rib thin-walled stiffened structure increased gradually with the increase of temperature. Fundamental frequency will reach the lowest point, as long as the temperature is around the buckling temperature. The above analysis shows that the stiffness of cross rib thin-walled stiffened structure changed with temperature. In the process of persistently increasing buckling temperature, the structure experienced the process of stability, instability and stability.

\section{References}

[1] Hieken M H. Sonic Fatigue Test Methods at Elevated Temperatures[R]. 1973.

[2] Hoff N J. Buckling at high temperature[J]. Journal of the Royal Aeronautical Society, 1957, 61(563): 756-774.

[3] Chen Rui Xi and Mei Chuh. Finite element nonlinear random response of beams to acoustic and thermal loads applied simultaneously[C]. AIAA/ASME/ASCE/AHS/ASC Structures, Structural Dynamics and Materials Conference, 34th 1993

[4] Ng Chung Fai. Design guide for predicting nonlinear random response(including snap-through) of buckled plates[J]. 1989

[5] Sha Y D, Wei J, Gao Z J, et Nonlinear Response of Snap-Through and Fatigue Life Prediction for Panels to Thermo-acoustic Loadings. Journal of Vibration and Control accepted 2012.

[6] Sha Y D, Gao Z J, Xu F nfluences of Thermal Loads on Nonlinear Response of Thin-Walled Structures in Thermo-Acoustic Environment [J]. Applied Mechanics and Materials, 105: 220-226. 